\title{
CLOSED IMAGES OF THE WALLMAN COMPACTIFICATION
}

\author{
DOUGLAS HARRIS
}

\begin{abstract}
The class of closed canonical images of the Wallman compactification of a space is given an internal characterization, and the class of locally compact-small spaces is introduced and shown to play for such compactifications the same role that locally compact spaces play for Hausdorff compactifications.
\end{abstract}

1. Introduction. Among Hausdorff spaces perhaps the next most interesting class of spaces after the class of compact spaces is the class of locally compact spaces. A large portion of this interest is due to the manner in which locally compact spaces are related to their compactifications. A number of conditions stated in terms of compactifications are known to be equivalent to local compactness.

The class of $T_{1}$-compactifications of a space never has a simple structure, even if the space being considered is compact. Thus hypotheses restricting the type of compactification considered are required in order to obtain structure theorems analogous to those that hold for $T_{1}$-compactifications. A class of $T_{1}$-compactifications that is well-behaved in this regard is the class of $\mathscr{W} \mathscr{C}$-compactifications, which are the canonical closed images of the Wallman compactification. This class of compactifications includes (in fact is identical with, as will be shown) $\omega \alpha$-compactifications of Osmatesku ([3], [4]), the class of Shanin compactifications [5], and all $T_{2}$-compactifications. The restriction seems especially natural as an analogue of the situation for $T_{2}$ spaces in which every compactification is a closed canonical image of the Stone-Cech compactification.

It will be shown that the locally compact-small spaces defined below play for $T_{1}$ spaces and $\mathscr{W} \mathscr{C}$-compactifications the same role that locally compact spaces play for $T_{2}$ spaces and compactifications. In particular it will be shown that the following five conditions are equivalent: the space $X$ is locally compact-small; the Alexandrov compactification is a $\mathscr{W} \mathscr{C}$ compactification; there is a one-point $\mathscr{W} \mathscr{C}$-compactification; there is a projectively smallest $\mathscr{W} \mathscr{C}$-compactification; the space $X$ is open in every $\mathscr{W} \mathscr{C}$-compactification; the space $X$ is open in its Wallman compactification. It will also be shown that every locally compact space is locally

Received by the editors October 27, 1972 and, in revised form, March 5, 1973. AMS (MOS) subject classifications (1970). Primary 54D45, 54D35. 
compact-small, and that the converse holds for $T_{3}$ but not for $T_{2}$ spaces. In addition an internal characterization of $\mathscr{W} \mathscr{C}$-compactifications will be given.

The term space shall mean a $T_{1}$ topological space, and map means a continuous function between spaces. The terminology and notation of [2] will be adopted throughout.

2. Locally compact-small spaces. A space is locally compact (in this paper) if every point has a compact neighborhood, and a space is locally compact-small if every point has a compact-small neighborhood, where a subset is compact-small if every closed set that it contains is compact.

2.1. Every locally compact space is locally compact-small.

2.2. A space is locally compact-small if and only if every neighborhood of every point contains a compact-small neighborhood.

The preceding result shows that local compact-smallness is an improvement on local compactness in that the analogue of 2.2 does not hold for local compactness.

2.3. A regular space is locally compact if and only if it is locally compactsmall.

Proof. If the space is locally compact-small then every point has a closed compact-small neighborhood, thus clearly a compact neighborhood.

2.4. CountereXAMple. A locally compact-small $T_{2}$ space need not be locally compact. Let $X$ be the real line, with basic neighborhoods of each $x \neq 0$ the usual Euclidean neighborhoods, and basic neighborhoods of $0 \in X$ the sets $V_{m}=\{x:-1 / m<x<1 / m, x \neq 1 / n$ for any $n\}$, for positive integers $m$. Then every point other than 0 has a compact neighborhood, and each $V_{m}$ is a compact-small neighborhood of 0 , for the subsets of $V_{m}$ that are closed in $X$ are closed in the usual topology on $X$, and thus are compact, since the subspace topologies are the same.

Another example will be given in 2.7 below.

2.5. Every closed subspace of a locally compact-small space is locally compact-small.

2.6. COUNTEREXAMPLE. An open subspace of a locally compact-small space need not be locally compact-small, for example, the rationals as a subspace of its Alexandrov compactification.

2.7. Counterexample. A locally compact-small space need not be a $k$-space. Let $Y$ be the Katětov extension of the countable discrete space $N$; 
specifically, points of $N$ are isolated points in $Y$, and a base for neighborhoods of $p \in Y-N$ is the collection $\{V \cup\{p\}\}$, where $V \subset N$ belongs to the ultrafilter $p$. Certainly each point of $N \subset Y$ has a compact-small neighborhood. Now if $p \in Y-N$ and $A$ is closed in $Y$ with $A \subset N \cup\{p\}$, then $A$ is finite, for if $A$ is infinite then $A \cap N$ is infinite and thus $A \cap N$ belongs to infinitely many free ultrafilters on $N$, each of which must belong to the closed superset $A$ of $A \cap N$. Thus $N \cup\{p\}$ is a compact-small neighborhood of $p \in Y-N$, so $Y$ is locally compact-small. To see that $Y$ is not a $k$-space, note that every compact subset of $Y$ is finite, so that every subset intersects every compact subset in a closed set.

3. $\mathscr{W} \mathscr{C}$-compactifications. The basic compactification to be considered is the Wallman compactification $(w X, w)$, the construction of which is given in $[6,17.17]$. A compactification $(Z, g)$ of $X$ is a $\mathscr{W} \mathscr{C}$ compactification if there is a closed map $h$ such that $h w=g$; equivalently the map $g: X \rightarrow Z$ is a $\mathscr{W} \mathscr{C}$-map as defined in [1] and [2]. We also define $(Z, g)$ to be a $\mathscr{W} O$-compactification if $g$ is a $\mathscr{W} \mathcal{O}$-map as defined in [2], and a $\mathscr{W}$-compactification if $g$ is a $\mathscr{W}$-map as defined in [2].

3.1. If $(Z, g)$ and $(Y, h)$ are $\mathscr{W} \mathscr{C}$-compactifications of $X$ then there is at most one map $m$ such that $m g=h$. Such a map is closed, and $m[Z-g[X]]=$ $Y-h[X]$.

Proof. Suppose $m g=h=n g$. There are closed maps $p: w X \rightarrow Z$ and $q: w X \rightarrow Y$ such that $p w=g$ and $q w=h$. Then $q w=m p w=n p w$ and since $w$ is an epimorphism (see [1]) it follows that $q=m p=n p$. Since $p$ is a closed map whose image is dense in its codomain, it is a surjection and therefore $m=n$. Since $q=m p$ is a closed map and $p$ is a surjection, it follows that $m$ is a closed map. Now if $z \in Z-g[X]$ then $z=p(s)$ for some $s \in w X-$ $w[X]$, and $m(z)=m p(s)=q(s)$. Since the trace filter of $q(s) \in Y$ on $X$ must be contained in the trace filter of $s \in w X-X$ on $X$, it follows that $q(s) \notin h[X]$.

\subsection{Every $\mathscr{W} \mathscr{C}$-compactification of $X$ has cardinal bounded by $2^{2^{\operatorname{crdn} x}}$}

Proof. Such a compactification is the image of $w X$, which as a strict relatively $T_{1}$ filter extension of $X$ has cardinal bounded as stated.

The $\mathscr{W} \mathscr{C}$-compactifications of a given space are ordered by $(Z, g) \geqq$ $(Y, h)$ if there is $m$ such that $m g=h$. It follows from 3.1 that this is a partial ordering.

3.3. Let $(Z, g)$ be a $\mathscr{W} \mathscr{C}$-compactification of $X$, and let $A \subset Z-g[X]$ be a closed set. Let $p: Z \rightarrow Y$ be the quotient map obtained by identifying $A$ to be a single point. Then $(Y, p g)$ is a $\mathscr{W} \mathscr{C}$-compactification of $X$.

Proof. The map $p$ is a closed map, thus $p g$ is a $\mathscr{W} \mathscr{C}$-map.

As an immediate corollary there is the following result. 
3.4. If $(Z, g)$ is a $\mathscr{W} \mathscr{C}$-compactification of $X$ such that $Z-g[X]$ has more than one point, then there is a $\mathscr{W} \mathscr{C}$-compactification $(Y, h)$ such that $(Z, g)>(Y, h)$.

The preceding results lead us to consider one-point compactifications of $X$. The Alexandrov compactification $(a X, a)$ (see $[6,17.1])$ is the largest of these; the smallest is obtained by adjoining to an infinite space a single finite complement point.

The space $X$ is open in the compactification $(Z, g)$ if $g[X]$ is open in $Z$; the compactification $(Z, g)$ is compact-closed if $g[K]$ is closed in $Z$ whenever $K$ is closed and compact in $X$.

3.5 The Alexandrov compactification is the unique one-point compactclosed compactification.

3.6. Let $(Z, g)$ be a compactification of $X$. There is a closed map $m: Z \rightarrow a X$ such that $m g=a$ and $m[Z-g[X]]=a X-a[X]$ if and only if $X$ is open in $(Z, g)$ and $(Z, g)$ is compact closed.

Proof. Suppose such a closed map $m$ exists. Then certainly $X$ is open in $(Z, g)$. If $K$ is closed and compact in $X$ then $m g[K]$ is closed in $a X$. Now $m\left[\mathrm{cl}_{Z} g[K]\right] \subset \mathrm{cl}_{Z} m g[K]=m g[K] \subset a[X]$; thus since $m[Z-g[Z]] \subset a X-a[X]$ it follows that $\mathrm{cl}_{Z} g[K]=g[K]$.

If $X$ is open in $(Z, g)$ and $(Z, g)$ is compact closed then it is readily seen that the quotient space obtained by identifying $Z-g[X]$ to a single point is the Alexandrov compactification.

3.7. REMARK. The conditions that $m$ is closed and that $m$ preserves the outgrowth are independent, and neither can be removed.

3.8. Every $\mathscr{W} \mathscr{C}$-compactification is a compact-closed compactification.

Proof. It is well known that $(w X, w)$ is a compact-closed compactification and it is clear that a closed mapping of compactifications preserves the property.

3.9. The Alexandrov compactification is the only possible one-point $\mathscr{W} \mathscr{C}$-compactification.

3.10. THEOREM. The following conditions are equivalent for a noncompact space $X$.

(a) The space $X$ is locally compact-small.

(b) The Alexandrov compactification is a $\mathscr{W} \mathscr{C}$-compactification.

(c) There is a smallest $\mathscr{W} \mathscr{C}$-compactification.

(d) There is a one-point $\mathscr{W} \mathscr{C}$-compactification.

(e) The space $X$ is open in some $\mathscr{W} \mathscr{C}$-compactification.

(f) The space $X$ is open in every $\mathscr{W} \mathscr{C}$-compactification.

(g) The space $X$ is open in its Wallman compactification. 
Proof. Trivially (b) implies (e), and it is readily shown from 3.1 that (e), (f), and (g) are equivalent. Using 3.6 it can be seen that (f) implies (c), and from 3.4 it follows that (c) implies (d). According to 3.9, (d) is equivalent to (b). Thus it remains only to show that (a) and (g) are equivalent.

If $X$ is locally compact small and $x \in X$, choose a compact small neighborhood $V$ of $x$. Clearly $V \subset w X-\mathrm{cl}_{w X}(X-V)$. Now if $p \in w X-$ $\mathrm{cl}_{w X}(X-V)$ there is a closed subset $B$ of $X$ such that $p \in \operatorname{cl}_{w X} B$ and $\mathrm{cl}_{w X} B \cap \mathrm{cl}_{w X}(X-V)=\varnothing$. It follows that $B \subset V$ and thus $B$ is compact, so $\mathrm{cl}_{w X} B=B$ and $p \in X$. Now $X-\mathrm{cl}_{w X}(X-V)=V$; thus $p \in V$. Therefore $V=w X-\mathrm{cl}_{w X}(X-V)$, so $V$ is open in $w X$.

If $X$ is open in $w X$ and $x \in X$, choose a closed set $B \subset X$ so that $x \notin \operatorname{cl}_{w X} B$ and $\operatorname{cl}_{w X} B \supset w X-X$. Now if $A$ is closed in $X$ and $A \cap B=\varnothing$ it follows that $\mathrm{cl}_{w X} A \cap \operatorname{cl}_{w X} B=\varnothing$ and thus $\mathrm{cl}_{w X} A \subset X$, so $A=\mathrm{cl}_{w X} A$ and $A$ is compact. Therefore the set $V=X-B$ is a compact small neighborhood of $x$.

3.11. REMARK. There are one-point compactifications with weaker topology than that of $(a X, a)$; these are all images of $(w X, w)$ whenever $(a X, a)$ is such an image, but $(a X, a)$ will be the only $\mathscr{W} \mathscr{C}$-compactification. When $X$ satisfies appropriate conditions such a smaller compactification $(Z, g)$ may in fact be a $\mathscr{W O}$-compactification; that is, the embedding $g: X \rightarrow Z$ may be a $\mathscr{W O}$-map as defined in [2]. Note also that whenever $(a X, a)$ is a $\mathscr{W} \mathscr{C}$-compactification (that is, the map $a: X \rightarrow a X$ is a $\mathscr{W}$-map as defined in [2]) then $X$ is open in $w X$ and thus $(a X, a)$ is a $\mathscr{W} \mathscr{C}$-compactification.

4. An internal characterization. It will be shown that the $\mathscr{W} \mathscr{C}$ compactifications of a space are those compactifications $(Z, g)$ satisfying the following condition.

$\mathscr{W} \mathscr{C}$. If $C$ and $D$ are closed in $Z$ and disjoint then there is a closed $B \subset X$ with $C \subset \operatorname{cl} g[B] \subset Z-D$, such that if $E$ is closed in $X$ and disjoint from $B$ then $\operatorname{cl} g[E] \cap C=\varnothing$.

Note that this condition is closely related to the definition given in [2] of a $\mathscr{W O}$-map. This is to be expected, since $\mathscr{W} \mathscr{C}$-compactifications are $\mathscr{W} \mathcal{O}$-compactifications.

\subsection{THEOREM. Every $\mathscr{W} \mathscr{C}$-compactification satisfies condition $\mathscr{W} \mathscr{C}$.}

Proof. Let $h: w X \rightarrow Z$ be the closed extension of $g: X \rightarrow Z$. Let $C, D \subset Z$ be closed and disjoint: Then $h^{-}[C]$ and $h^{-}[D]$ are closed and disjoint in $w X$, so by $[2,2.9]$ there is a closed $B \subset X$ with $h^{\leftarrow}[C] \subset \mathrm{cl}_{w X} w_{X}[B]$ and $\mathrm{cl}_{w X} w_{X}[B] \cap h^{-}[D]=\varnothing$. Then

$$
C=h\left[h^{\leftarrow}[C]\right] \subset h\left[\mathrm{cl}_{w X} w_{X}[B]\right]=\mathrm{cl}_{Z} h_{w_{X}}[B]=\mathrm{cl}_{Z} g[B]
$$

(since $h$ is a closed onto map) and $\operatorname{cl}_{Z} g[B]=g\left[\mathrm{cl}_{w X} w_{X}[B]\right] \cap D=\varnothing$. 
If $E$ is closed in $X$ and disjoint from $B$ then $\operatorname{cl}_{w X}[E] \cap \mathrm{cl}_{w X} w_{. X}[B]=\varnothing$, thus $\mathrm{cl}_{w X}[E] \cap g^{-}[D]=\varnothing$, so $\mathrm{cl}_{Z} g[E] \cap D=g\left[\mathrm{cl}_{w X} w_{X}[E]\right] \cap D=\varnothing$.

We now turn our attention to the converse. That is, we show that every compactification that satisfies condition $\mathscr{W} \mathscr{C}$ is a $\mathscr{W} \mathscr{C}$-compactification.

4.2. Every compactification that satisfies condition $\mathscr{W} \mathscr{C}$ is a strict topological extension.

Proof. Let $z \in Z$ and let $V$ be an open neighborhood of $z$. Taking $C=Z-V$ and $z=D$ and choosing $B$ as in condition $\mathscr{W} \mathscr{C}$ it follows that $z \in Z-\mathrm{Cl}_{Z} g[B] \subset V$.

4.3. Every compactification that satisfies condition $\mathscr{W} \mathscr{C}$ is a $\mathscr{W} \mathfrak{C}$ compactification.

Proof. We must show that $g: X \rightarrow Z$ is a $\mathscr{W} \mathcal{O}$-map. Let $v$ be an open cover of $Z$. For each $z \in Z$ choose $V \in \nu$ such that $z \in V$, and using condition $\mathscr{W} \mathscr{C}$ choose a closed subset $A_{z}$ of $X$ with $z \in Z-\mathrm{cl}_{Z} g\left[A_{z}\right] \subset V$, such that if $E \subset X$ is closed and disjoint from $A_{z}$ then $\mathrm{cl}_{Z} E \subset V$. Since $Z$ is compact, there is a finite collection $A_{1}, \cdots, A_{n}$ of the $A_{z}$ such that $\left\{Z-\operatorname{cl}_{Z} g\left[A_{i}\right]\right\}$ covers $Z$. In particular $\mu=\left\{X-A_{i}\right\}$ covers $X$. It now follows that $\mu<g \nu$. Thus $g$ is a $\mathscr{W} \mathcal{O}$-map.

The following condition on compactifications is useful in the present and many other contexts.

$\mathscr{F} \mathscr{C}$. If $A \subset X$ is closed and $z \in \mathrm{cl}_{Z} g[A]$ then there is a closed filter on $X$ that contains $A$ and has $z$ as its unique cluster point in $Z$.

\subsection{Every compactification that satisfies $\mathscr{W} \mathscr{C}$ satisfies $\mathscr{F} \mathscr{C}$.}

Proof. Since $z \in \mathrm{cl}_{Z} g[A]$, then the principal filter generated by $A$ is a closed filter on $X$ that contains $A$ and has $z$ as cluster point in $Z$. Thus there exists a closed filter $\lambda$ on $X$ that contains $A$, has cluster point $z \in Z$, and is maximal with respect to these two properties. We shall show that $\lambda$ has no cluster point in $Z$ other than $z$. Suppose $y \in Z$ with $y \neq z$. Using $\mathscr{W} \mathscr{C}$ there is a closed $B \subset X$ such that $z \in \mathrm{cl}_{Z} g[B] \subset Z-y$ with the property that if $C \subset X$ is closed with $C \cap B=\varnothing$ then $z \notin \mathrm{cl}_{Z} g[C]$. It follows immediately that $B$ intersects every member of $\lambda$, and since $z \in \mathrm{cl}_{Z} B$ then $B \in \lambda$. Thus $y$ is not a cluster point of $\lambda$.

The next condition is due to Osmatesku [3], who defines $\omega \alpha$-compactifications as those $\mathscr{W}$-compactifications that satisfy the condition.

$\mathscr{K} \mathscr{C}$. If $A \subset X$ is closed and $k \subset Z$ is compact with $g[A] \subset K \subset \operatorname{cl} g[A]$ then $K$ is closed.

4.5. Every compactification that satisfies $\mathscr{F} \mathscr{C}$ (thus those that satisfy $\mathscr{W} \mathscr{C}$ ) satisfies $\mathscr{K} \mathscr{C}$. 
Proof. Let $z \in \operatorname{cl} g[A]$. Then by $\mathscr{F} \mathscr{C}$ there is a closed filter $\lambda$ on $X$ that contains $A$ and has $z$ as its unique cluster point in $Z$. Since $k$ is compact, $g(\lambda)$ must have a cluster point in $k$, and thus $z \in K$.

The following result now completes the proof of the converse of 4.1. It also establishes the equivalence of $\mathscr{W} \mathscr{C}$-compactifications with $\omega \alpha$ compactifications mentioned in the introduction.

4.6. Every $\mathscr{W}$-compactification (hence every $\mathscr{W} O$-compactification) that satisfies $\mathscr{K} \mathscr{C}$ is a $\mathscr{W} \mathscr{C}$-compactification.

ProOF. Let $h: w X \rightarrow Z$ with $h w^{X}=g$. If $A \subset X$ is closed then $A \subset h\left[\mathrm{cl}_{w X} w_{X}[A]\right] \subset \mathrm{cl}_{Z} g[A]$. Since $g\left[\mathrm{cl}_{w X} w_{X}[A]\right]$ is compact then by $\mathscr{K} \mathscr{C}$ it is closed, hence equal to $\mathrm{cl}_{Z} g[A]$. Now let $B \subset Z$ be closed and set $\mathscr{B}_{B}=\left\{A \subset X: A\right.$ is closed and $\left.B \subset \mathrm{cl}_{w X} w_{X}[A]\right\}$. Since $\mathrm{cl}_{w X} w_{X}[A] \cap$ $\mathrm{cl}_{w X} w_{X}[C]=\operatorname{cl}_{w X} w_{X}[A \cap C]($ by $[2,2.4])$ it follows that $\mathscr{B}_{B}$ is a closed filter base on $X$. Since $w X$ is a strict extension of $X$, it follows that $B$ is precisely equal to the set of cluster points in $w X$ of $\mathscr{B}_{B}$. Now clearly $h[B]$ is contained in the closed set $D$ of cluster points of the filterbase $h\left(\mathscr{B}_{B}\right)$. Now if $z \in D$ then (since $\left.h\left[\mathrm{cl}_{w X} w_{X}[A]\right]=\mathrm{cl}_{Z} g[A]\right) h^{\leftarrow}(z) \cap$ $\operatorname{cl}_{w X} w_{X}[A]=\varnothing$ for each $A \in \mathscr{B}_{B}$, and thus $\mathscr{B}_{B}$ has a cluster point $p \in h^{-}(z)$. It follows that $z=h(p) \in h[B]$. We have shown that $h[B]=D$, thus $h$ is a closed map.

The following two results have now been established.

4.7. THEOREM. Every compactification that satisfies $\mathscr{W} \mathscr{C}$ is a $\mathscr{W} \mathscr{C}$ compactification.

4.8. THEOREM. A compactification satisfies $\mathscr{W} \mathscr{C}$ if and only if it is $\omega \alpha$-compactification.

4.9. Remark. Osmatesku gives a condition in [4], stated in terms of proximites, that is closely related to $\mathscr{W} \mathscr{C}$. The condition (modified slightly) is that if $F, G \subset X$, with $\operatorname{cl}_{Z} g[F] \cap \operatorname{cl}_{Z} g[G]=\varnothing$, then there is $B \subset X$ such that $\mathrm{cl}_{Z} g[F] \subset \mathrm{cl}_{Z} g[B], \mathrm{cl}_{Z} g[G] \cap \mathrm{cl}_{Z} g[B]=\varnothing$, and if $E \subset X$ is closed with $\operatorname{cl}_{Z} g[B \cap E] \cap \operatorname{cl}_{Z} g[F]=\varnothing$ then $\operatorname{cl}_{Z} g[E] \cap \operatorname{cl}_{Z} g[F]=\varnothing$. Then he shows that the $\omega \alpha$-compactifications are the strict compactifications satisfying this condition. Note that Osmatesku's condition trivially implies ours for $C$ and $D$ of the form $\mathrm{cl}_{Z} g[G], \mathrm{cl}_{Z} g[F]$, since if $B \cap E=\varnothing$ then certainly $\mathrm{cl}_{Z} g[B \cap E] \cap \mathrm{Cl}_{Z} g[F]=\varnothing$, so $\mathrm{cl}_{Z} g[E] \cap \mathrm{cl}_{Z} g[F]=\varnothing$. Also note that our condition may be restricted to sets $C$ and $D$ of this special form if we add the condition that $(Z, g)$ is a strict extension.

One additional condition on compactifications is of special interest.

$\mathscr{C} \mathscr{B}$. There is a base $\mathscr{B}$ for closed sets in $X$ such that cl $g(\mathscr{B})=\{\mathrm{cl} g[B]$ : $B \in \mathscr{B}\}$ is a base for closed sets in $Z$, and $\operatorname{cl} g[B] \cap \operatorname{cl} g[C]=\operatorname{cl} g[B \cap C]$ for $B, C \in \mathscr{B}$. 


\subsection{Condition $\mathscr{C} \mathscr{B}$ implies $\mathscr{W} \mathscr{C}$.}

Proof. Let $C$ and $D$ be disjoint closed subsets of $Z$. Since $Z$ is compact and $\mathrm{cl} g(\mathscr{B})$ is a base for closed sets in $Z$ then there is $B \in \mathscr{B}$ with $C \subset$ $\operatorname{cl} g[B]$ and $\operatorname{cl} g[B] \cap D=\varnothing$. Now if $E \subset X$ is closed then $\operatorname{cl} g[E] \cap c l g[B]=$ $\operatorname{cl} g[E \cap B]$ (since $B \in \mathscr{B}, \operatorname{cl} g(\mathscr{B})$ is a base for $Z$, and $\operatorname{cl} g[A \cap B]=\operatorname{cl} g[A] \cap$ $\operatorname{cl} g[B]$ for all $A \in \mathscr{B})$. Thus if $E \cap B=\varnothing$ then $\operatorname{cl} g[E] \cap \operatorname{cl} g[B]=\varnothing$, so $\operatorname{cl} g[E] \cap C=\varnothing$.

A Shanin compactification (see [5]) is a compactification for which $\mathscr{C} \mathscr{B}$ is satisfied by a base $\mathscr{B}$ that contains the empty set and is closed under finite union. The following result is thus a special case of 4.10.

4.11. THEOREM. Every Shanin compactification is a $\mathscr{W} \mathscr{C}$-compactification.

\section{REFERENCES}

1. D. Harris, The Wallman compactification is an epireflection, Proc. Amer. Math. Soc. 31 (1972), 265-267. MR 44 \#5927.

2. - The Wallman compactification as a functor, General Topology and Appl. 1 (1971), 273-281. MR 45 \#1122.

3. P. K. Osmatesku, wa-compactifications, Vestnik. Moskov. Univ. Ser. I Mat. Meh. 1963, no. 6, 45-54. MR 28 \#579.

4. —, Proximities on $T_{1}$-spaces, Czechoslovak Math. J. 19 (94) (1969), 193-207. (Russian) MR 40 \#1986.

5. N. A. Sanin, On the theory of biocompact extensions of topological spaces, C.R. (Dokl.) Acad. Sci. URSS 38 (1943), 154-156. MR 5, 46.

6. W. Thron, Topological structures, Holt, Rinehart and Winston, New York, 1966. MR 34 \#778.

Department of Mathematics, Marquette University, Milwaukee, Wisconsin 53223 